\title{
Linear off null working condition for total internal reflection imaging ellipsometry to detect subtle electron density change
}

Jin, Xiangnan, Wang, Chenyu, Shen, Jia, Liu, Wei, Luo, Ziren, et al.

Xiangnan Jin, Chenyu Wang, Jia Shen, Wei Liu, Ziren Luo, Yu Niu, "Linear off null working condition for total internal reflection imaging ellipsometry to detect subtle electron density change," Proc. SPIE 11781, 4th Optics Young Scientist Summit (OYSS 2020), 117811I (28 February 2021); doi: 10.1117/12.2591323

SPIE. Event: Optics Frontier: Optics Young Scientist Summit, 2020, Ningbo, China 


\title{
Linear off null working condition for total internal reflection imaging ellipsometry to detect subtle electron density change
}

\author{
Xiangnan Jin ${ }^{\mathrm{a}^{*}}$, Chenyu Wang ${ }^{\mathrm{b}, \mathrm{c}^{*}}$, Jia Shen ${ }^{\mathrm{b}, \mathrm{c}}$, Wei Liu ${ }^{\mathrm{d} * *}$, Ziren Luo ${ }^{\mathrm{d}} \& \mathrm{Yu} \mathrm{Niu}^{\mathrm{d}}$ \\ ${ }^{a}$ School of Fundamental Physics and Mathematical Sciences, Hangzhou Institute for Advanced \\ Study, University of Chinese Academy of sciences, 1 Xiangshan Branch Alley, Hangzhou, 310024, \\ China; \\ ${ }^{b}$ NML, Beijing Key Laboratory of Engineered Construction and Mechanobiology, Institute of \\ Mechanics, Chinese Academy of Sciences, 15 Bei-si-huan West Road, Beijing, 100190, China; \\ ${ }^{\mathrm{c}}$ School of Engineering Science, University of Chinese Academy of Science, 19 Yu-quan Road, \\ Beijing, 100049, China; \\ ${ }^{\mathrm{d}}$ Center for Gravitational Wave Experiment, Institute of Mechanics, Chinese Academy of Science, \\ 15 Bei-si-huan West Road, Beijing, 100190, China
}

*These authors contributed equally to this work

**Corresponding author: email: liuwei@imech.ac.cn

\begin{abstract}
Total internal reflection imaging ellipsometry (TIRIE) is widely used in the field of the biological detection due to its high sensitivity and multi-detection capability. Traditionally, the ellipsometric measurement works under the null-off null condition which is insensitive to the small interface variations such as the electron density disturbance at the sensing surface. Thus, we analyze the response of the detected signals under the different working conditions to the ellipsometric parameter variations and optimize the polarization settings to further enhance the TIRIE response to the subtle interface variation in this paper. Furthermore, the relationship between the detected signal and the electron density disturbance is obtained, and the result shows that the detection sensitivity for the subtle interface changes is improved by one hundred times under the optimized working condition.
\end{abstract}

Keywords: TIRIE, working conditions, electron density

\section{INTRODUCTION}

Total internal reflection imaging ellipsometry (TIRIE) is a phase sensitive surface plasmon resonance imaging (SPRi) biosensor which combines SPR with imaging ellipsometry (IE) technology. It is a real-time multi-detection approach to visualize the biomolecule interactions at the interface. Compared with SPR biosensors which measure the intensity variation of the reflection, IE provides an extra ellipsometric phase sensitivity. Traditionally, an ellipsometry-based biosensor prefers to work under the null-off null condition ${ }^{1}$, which requires the light extinction by adjusting the azimuths of the polarizer and the analyzer at the initial, so called the null condition and then, when the optical thickness of the interface changes, some reflection will pass through the fixed analyzer, arousing the detected light intensity increasing in detector. This preference comes from the fact that the null condition is taken as the zero point of the ellipsometry system at which the system error is well depressed and the ellipsometric parameters of the surface can be determined ${ }^{2}$. Further, the null condition is easy to calibrate: finding a minimal in the signal of the detector by rotating the polarizer and the analyzer. However, this null-off null condition might not suitable for the small interface variations at the sensing surface in the neighborhood of the null condition, as is pointed by Hans Arwin ${ }^{1}$, the detected signal responds to the variations of the ellipsometric parameters quadratically. Further, for the sensing applications interested in the relative change in the interface rather than the absolute ellipsometric parameters, a linear response is favorable, especially for small interface variations.

A popular strategy to amplify the response of the optical biosensor is to introduce nanomaterials for the large specific surface area and the local surface plasmon resonance enjoyed by the precious metal nanoparticles. The strategy 
significantly improving the sensitivity of the sensors in the detection of the small molecules ${ }^{3,4,5}$. However, the repeatability of the experimental conditions is not ideal due to the poor uniformity and reproductivity caused by the preparation of the nanomaterials.

To further increase the sensitivity of a polarizer-compensator-sample-analyzer (PCSA) ellipsometer biosensor, the optimal azimuth angle settings are studied ${ }^{6,7}$ corresponding to the interface change. For example, Yanyan Chen established a linear relationship between the detected signal and the thickness of $\mathrm{SiO}_{2}$ layer on a silicon chip instead of the traditional nonlinear one ${ }^{8}$. However, no optimization for TIRIE biosensor is discussed, especially for the small interface variations such as the electron density disturbance at the sensing surface.

In this paper, we will briefly review the null-off null working condition and then discuss the optimized off-null working condition for TIRIE biosensor. Finally, a comparation between the two conditions is discussed for the subtle surface electron disturbance.

\section{OPTIMIZATION OF WORKING CONDITIONS}

The structure of TIRIE biosensor based on a PCSA configuration is shown in Figure 1, which consists of an xenon light source, a polarizer, a compensator (quarter wave plate), a sensing surface, an analyzer and a CCD, where the sensing surface is a $48 \mathrm{~nm}$ gold film deposited on SF 10 glass. The light beam transforms into the elliptically polarized light after passing through the polarizer and the compensator, perpendicularly falls to the prism and finally is reflected by the sensing surface. As the incident angle is larger than the critical angle of the sensing surface, the incident light is totally reflected after it reaches the sensing surface, along with the evanescent wave the amplitude of which decays exponentially. Due to the existence of the evanescent wave, the polarization state of the reflected light contains the sample information on the sensing surface. After passing through the analyzer, the reflected light becomes the linearly polarized and captured by the CCD imaging system, and the image of the sensing surface is saved in the computer.

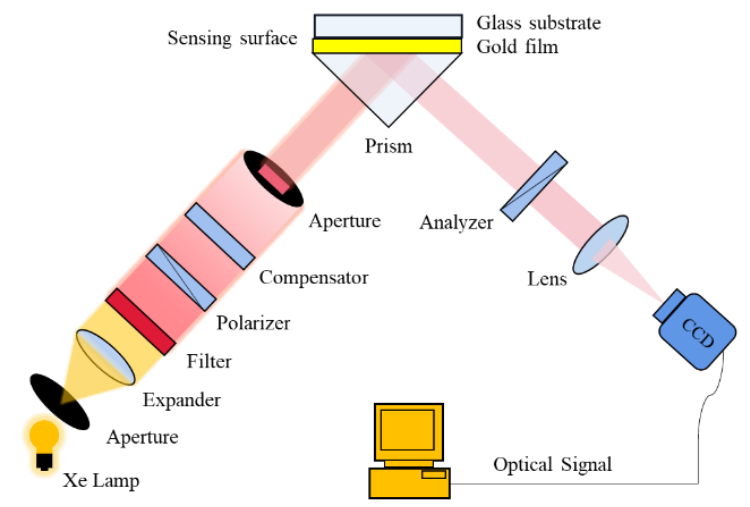

Figure 1. Schematic diagram of the TIRIE based on a PCSA configuration

By applying a Jones vector and matrix approach using the notation convention of R.M.A. Azzam and N.M. Bashara ${ }^{9}$ the expression for the detected intensity in a typical PCSA system is given by

$$
I=G L L^{*}
$$

where * denotes a complex conjugation, $G=\left|K_{p}\right|^{2}\left|K_{C}\right|^{2}\left|K_{A}\right|^{2} K_{D} \cdot K_{P}$ and $K_{A}$ contain information on the intensity and absolute phase of the wave emergent from the polarizer and the analyzer, respectively. $K_{C}$ accounts for the equal attenuation and phase shift along the fast and the slow axes of the compensator, and $K_{D}$ is the real factor that depends on the intensity profile of the light beam and the nature of the detector.

$$
\begin{aligned}
L= & R_{p} \cos A\left[\cos C \cos (P-C)-\rho_{C} \sin C \sin (P-C)\right] \\
& +R_{S} \sin A\left[\sin C \cos (P-C)+\rho_{C} \cos C \sin (P-C)\right]
\end{aligned}
$$


Here $\rho_{C}$ is the slow-to-fast relative complex-amplitude transmittance of the compensator, and $R_{p}$ and $R_{S}$ are the complex reflectance of the sample for light polarized parallel and perpendicular to the plane of incidence, respectively. For an ideal compensator, $\rho_{C}=-j=-\sqrt{-1}$ and its azimuth is fixed at $C=\frac{\pi}{4}$. Thus, eq. (2) yields

$$
I=G \frac{\left|R_{S}\right|^{2}}{4 \cos ^{2} \Psi}[1-\cos 2 \Psi \cos 2 A+\sin 2 \Psi \sin (2 P+\Delta) \sin 2 A]
$$

$\mathrm{Or}^{2}$

$$
I=G \frac{\left|R_{S}\right|^{2}}{2 \cos ^{2} \Psi}\left[\sin ^{2}(A-\Psi)+\sin 2 A \sin 2 \Psi \sin ^{2}\left(P+\frac{\Delta}{2}-\frac{3}{4} \pi\right)\right]
$$

where $\Psi$ and $\Delta$ are the ellipsometric parameters, defined as the complex reflectance ratio

$$
\rho=\frac{R_{p}}{R_{s}}=\tan \Psi e^{j \Delta}
$$

In order to get the explicit relationship between the detected light intensity and the ellipsometric parameters, theoretical analysis has been specifically deduced.

\subsection{The null-off null working condition}

As is seen in eq. (4), the null condition is fulfilled by $A=\bar{\Psi}$ and $P=\frac{3}{4} \pi-\frac{\bar{\Delta}}{2}$, where $\bar{\Psi}$ and $\bar{\Delta}$ are the unperturbed ellipsometric parameters. At the null condition, we have $I_{\text {null }}=0$.

Under the assumption that $R_{S}$ is insensitive to the interface variation, $\left|R_{\mathrm{S}}\right|^{2}$ can be taken as a constant. This assumption holds for TIRIE biosensor. For TIRIE biosensor, the adsorption of $1 \mathrm{~nm}$ protein at the gold sensing surface will also cause $\left|R_{\mathrm{S}}\right|^{2}$ vary $0.002 \%$ around SPR angle.

Thus, when the optical thickness varies at the interface, the ellipsometric parameters change and the detected signal is given by ${ }^{1}$

$$
\delta I=G \frac{\left|R_{S}\right|^{2}}{2 \cos ^{2} \bar{\Psi}}\left[(\delta \Psi)^{2}+\frac{1}{4} \sin ^{2} 2 \bar{\Psi}(\delta \Delta)^{2}\right]
$$

where $\delta \Psi$ and $\delta \Delta$ are the ellipsometric parameter variations.

\subsection{The linear off null working condition}

For our sensing applications, our IE biosensor often focuses on the relative change at the interface instead of the exact ellipsometric parameters, which implies the largest imaging contrast variation after the adsorption at the optimal azimuths of the polarizer and the analyzer. Further, a linear response is expected for the small variations. Thus, the Taylor expansion of eq. (3) is given by

$$
\begin{gathered}
\delta I=G \frac{\left|R_{s}\right|^{2}}{2 \cos ^{2} \Psi}[\tan \Psi+\cos 2 A \tan \Psi+\sin 2 A \sin (2 P+\Delta)] \delta \Psi \\
+G \frac{\left|R_{S}\right|^{2}}{4 \cos ^{2} \Psi} \sin 2 A \sin 2 \Psi \cos (2 P+\Delta) \delta \Delta
\end{gathered}
$$

In general, it is tedious to get the extremum of $\delta I$ at the optimal azimuths of the polarizer $(P)$ and the analyze $(A)$ analytically. Here, we would only focus on the situation that the angle of incidence fulfills $\delta \Psi \approx 0$, for as a phase-sensitive measurement, $\Delta$ plays a key role in certain circumstances. When $\delta \Psi \approx 0$,eq. (7) can be simplified as

$$
\delta I=G \frac{\left|R_{S}\right|^{2}}{4 \cos ^{2} \Psi} \sin 2 A \sin 2 \Psi \cos (2 P+\Delta) \delta \Delta
$$

Obviously, the maximum $\delta I=\frac{G}{2}\left|R_{S}\right|^{2} \tan \bar{\Psi} \delta \Delta$ can be achieved at $\mathrm{A}=\frac{\pi}{4}, P=\pi-\frac{\bar{\Delta}}{2}$ or $\mathrm{A}=\frac{3}{4} \pi, P=\frac{\pi}{2}-\frac{\bar{\Delta}}{2}$ and the minimum $\delta I=-\frac{G}{2}\left|R_{S}\right|^{2} \tan \bar{\Psi} \delta \Delta$ at $\mathrm{A}=\frac{\pi}{4}, P=\frac{\pi}{2}-\frac{\bar{\Delta}}{2}$ or $\mathrm{A}=\frac{3}{4} \pi, P=\pi-\frac{\bar{\Delta}}{2}$. 
Eq. (8) implies that at the angle of incidence fulfilling $\delta \Psi \approx 0$, the azimuth of the analyzer can be fixed at $\pi / 4$ or $3 \pi / 4$. The azimuth of the polarizer can be optimized for the bare substrate experimentally. At this condition, the detected signal will respond to the small interface variation linearly.

According to eq. (8), the detected signal variation, $\delta I$, is dependent on the device parameter $\mathrm{G}$, and the light source intensity which influences $\left|R_{S}\right|^{2}$, it is difficult to make a comparison for real application. Thus, a normalized indicator is needed.

At the linear off-null working condition, where $I_{0}=G \frac{\left|R_{S}\right|^{2}}{4 \cos ^{2} \bar{\Psi}}$, which is far from the null condition, at which $I_{\text {null }}=0$, we have

$$
\frac{\delta I}{I_{0}}=\sin 2 \bar{\Psi} \sin \delta \Delta
$$

For small variation, we have $\sin \delta \Delta \approx \delta \Delta$, thus

$$
\frac{\delta I}{I_{0}}=\sin 2 \bar{\Psi} \delta \Delta
$$

Eqs. (9a) and (9b) suggest $\delta I / I_{0}$ can be used as the normalized indicator for it is independent from the device and the light source and only determined by the angle of the incidence at which the variation of parameter $\Psi$ can be neglected.

\subsection{The comparation of two working conditions for TIRIE}

According to eqs. (6) and (9b), it is apparent that the relationship between the variations of the light intensity and the ellipsometric parameters is a quadratic function under the null-off null working position, while a linear relationship under the linear off null working condition. Since the transformation of the surface characterization is caused by the change of the dielectric constant, we use the variation of the metal surface electron density to compare the response under the two working conditions.

Since TIRIE takes a gold-covered substrate, the SPR angle can be estimated as ${ }^{10}$

$$
\sin \phi_{r}=\sqrt{\frac{\varepsilon_{1} \varepsilon_{2}}{\left(\varepsilon_{1}+\varepsilon_{2}\right) \varepsilon_{0}}}
$$

where $\varepsilon_{i}=n_{i}^{2}(i=0,1,2)$ is the dielectric constant of each medium.

Now we will analyze the ellipsometric response at the SPR angle. For a typical glass/gold/solution model, the ellipsometric response owing to the dielectric constant variations of the gold $\delta \varepsilon_{1}$ and the solution, $\delta \varepsilon_{2}$, can be given by ${ }^{11}$

$$
\begin{gathered}
\delta \psi=\left(\frac{1}{2} \sin 2 \psi\right) \operatorname{Re}\left(\kappa_{1} \delta \varepsilon_{1}+\kappa_{2} \delta \varepsilon_{2}\right)=\left(\frac{1}{2} \sin 2 \psi\right)\left[\operatorname{Re}\left(\kappa_{1}\left(\varepsilon_{1}-1\right)\right) \delta \tau+\operatorname{Re}\left(\kappa_{2}\right) \delta \varepsilon_{2}\right] \\
\delta \Delta=\operatorname{Im}\left(\kappa_{1} \delta \varepsilon_{1}+\kappa_{2} \delta \varepsilon_{2}\right)=\operatorname{Im}\left(\kappa_{1}\left(\varepsilon_{1}-1\right)\right) \delta \tau+\operatorname{Im}\left(\kappa_{2}\right) \delta \varepsilon_{2}
\end{gathered}
$$

in which $\delta \tau$ is the electron density change of the gold film. $\kappa_{1}$ and $\kappa_{2}$ demonstrates the extent of modulation of the reflection polarization by the tiny variation in the dielectric constant of the metal and the electrolyte respectively ${ }^{11}$. 
Based on results above, it is not hard to obtain the connection between the detected light intensity and the charge density disturbances. As is seen in Figure 2, three working conditions are compared when the surface electron density $\tau$ disturbs. The response to the disturbance is parabolic under the traditional null-off null condition and the maximum response for $\tau=10^{-3} \mu \mathrm{m}^{-2}$ is around $10^{-5}$, barely detectable (Figure $2 \mathrm{~b}$ ). On the other hand, the responses under the linear off null condition are around $10^{-3}, 100$ times larger than that under the null-off null condition. Under the optimal azimuth of the analyzer, $42.6^{\circ}$, the maximum response is twice than that of $\mathrm{A}=45^{\circ}$ (Figure 2a). However, the latter is convenient to calibrate. Thus, the azimuth of the analyzer is fixed at $\mathrm{A}=45^{\circ}$ for our applications.

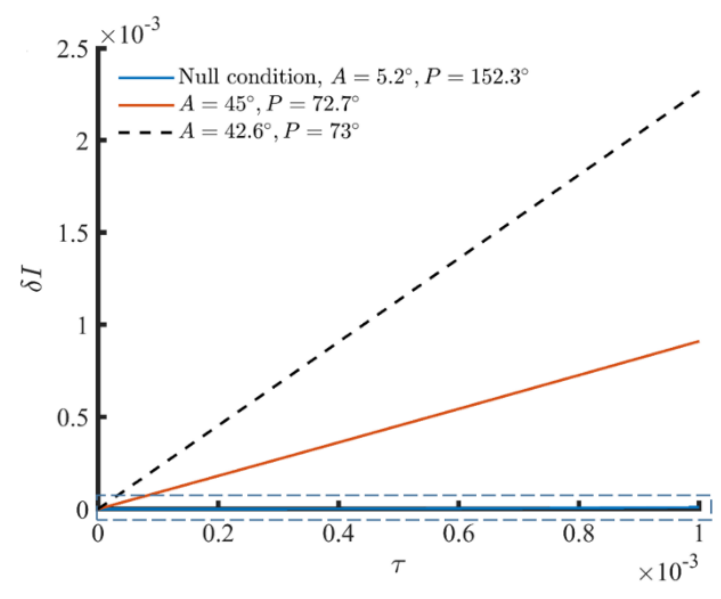

(a)

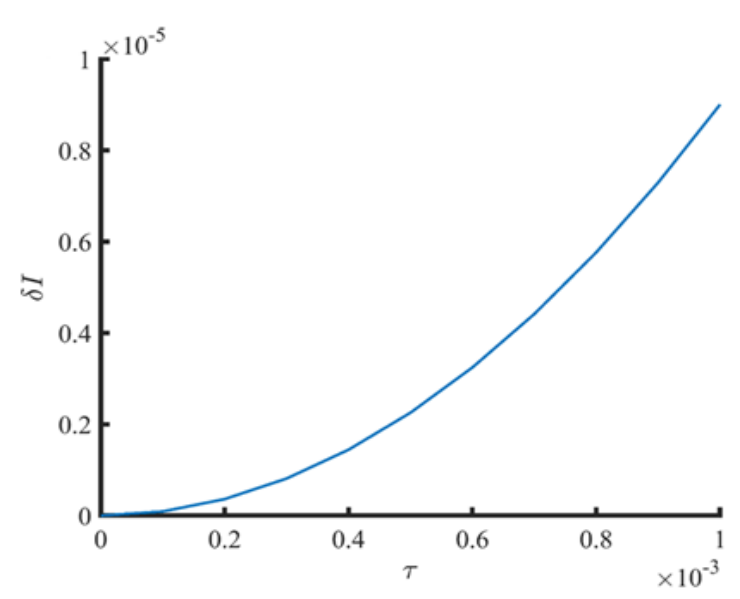

(b)

Figure 2. Working condition comparation for TIRIE biosensor when the surface electron density disturbs. (2a) Three working conditions for TIRIE biosensor: the null-off null working condition, the linear off-null working condition where the azimuth of analyzer is fixed at $45^{\circ}$ and the optimal linear off null condition where the azimuths of polarizer and analyzer are optimized to make the detected signal variation maximum. (2b) The enlarged null-off null working condition.

\section{CONCLUSION}

In this paper, we propose a linear off null working condition for TIRIE biosensor to detect small interface variation. At the expense of insensitivity to the ellipsometric amplitude, $\psi$, the detected signal gains extra phase sensitivity to the subtle surface change when the angle of incidence is fixed at the SPR angle. Further, the optimal azimuth angle of the analyzer is fixed at $\pi / 4$ or $3 \pi / 4$ will also simplify the optimization process. As a result, the detected the detected signal of TIRIE biosensor for subtle surface electron density variation is amplified 100 times under the linear off null working condition compared with the traditional null-off null working condition.

In general, the noise can also affect the sensitivity of TIRIE biosensor. At present, the dominant noise of the system is the power fluctuation of the light source. Meanwhile, the noise from the temperature fluctuation, the air disturbance and the ground vibration also have a non-negligible impact on the sensitivity of TIRIE. We will quantify the noise level and give the noise suppression plans in the future work, which are instructive for the applications of TIRIE.

\section{ACKNOWLEDGEMENT}

The authors acknowledge the support of the Open Fund of State Key Laboratory of Applied Optics (SKLAO2020001A01). 


\section{REFERENCES}

[1] Arwin, H., "Off-Null Ellipsometry Revisited: Basic Considerations for Measuring Surface Concentrations at Solid/Liquid Interfaces," Journal of colloid and interface science 156(2), 377-382 (1993).

[2] Azzam, R., Zaghloul, A.-R. \& Bashara, N., "Polarizer-surface-analyzer null ellipsometry for film-substrate systems," JOSA 65(12), 1464-1471 (1975).

[3] Wang, Z. L. et al., "Nanoparticles-Enabled surface-enhanced imaging ellipsometry for amplified biosensing," Analytical Chemistry 91(10), 6769 (2019).

[4] Li, Y. K., Liu, W., Jin, G., Niu, Y., Chen, Y. P., and Xie, M, X., "A Label-free sandwich imaging ellipsometry immunosensor for serological detection of procalcitonin," Analytical Chemistry 90(13), 8002 (2018).

[5] Paiva, T. O., Almeida, I., Marquês, J. T., Liu, W., Niu, Y., Jin, G., Viana, A. S., "Nanostructured interfaces with site-specific bioreceptors for immunosensing," Applied Surface Science 455, 412 (2017).

[6] Wang, G., Arwin, H., Jansson, R., "Optimization of azimuth angle settings in polarizer-compensator-sampleanalyzer off-null ellipsometry", Applied optics 42(1), 38-44 (2003).

[7] Wang, G., Arwin, H., Jansson, R., "Optimization of off null ellipsometry in sensor applications," Applied optics 43, 2000-2005 (2004).

[8] Chen, Y. Y., Meng, Y. H., Jin, G., "Optimization of off-null ellipsometry for air/solid interfaces," Applied optics 46(35), 8475-8481(2007).

[9] Azzam, R., and Bashara, N., [Ellipsometry and Polarized Light], North-Holland, New York, (1977).

[10] Raether, H., [Surface plasmons on smooth surfaces], Springer, (1988).

[11] Liu, W., Niu, Y., Viana, A. S., Correia, J. P. \& Jin, G., "Potential Modulation on Total Internal Reflection Ellipsometry," Analytical Chemistry 88(6), 3211-3217 (2016). 